\title{
Factors Controlling Oxygen Migration Barriers in Perovskites
}

Tam T. Mayeshiba

University of Wisconsin-Madison

1509 University Dr., Madison, Wisconsin, 53706

Dane D. Morgan*

University of Wisconsin-Madison

1509 University Dr., Madison, Wisconsin, 53706

ddmorgan@wisc.edu

\begin{abstract}
Perovskites with fast oxygen ion conduction can enable technologies like solid oxide fuel cells. One component of fast oxygen ion conduction is low oxygen migration barrier. Here we apply ab initio methods on over 40 perovskites to produce a database of oxygen migration barriers ranging from 0.2 to $1.6 \mathrm{eV}$. Mining the database revealed that systems with low barriers also have low metal-oxygen bond strength, as measured by oxygen vacancy formation energy and oxygen p-band center energy. These correlations provide a powerful descriptor for the development of new oxygen ion conductors and may explain the poor stability of some of the best oxygen conducting perovskites under reducing conditions. Other commonly-cited measures of space, volume, or structure ideality showed only weak correlation with migration barrier. The lowest migration barriers $(<0.5 \mathrm{eV})$ belong to perovskites with non-transition-metal B-site cations, and may require vacancycreation strategies that involve no dopants or low-association dopants for optimal performance.
\end{abstract}

\section{Introduction}

Fast oxygen migration is a critical property for technologies that involve oxygen transport and exchange with the environment,[1] including solid oxide fuel cells (SOFCs), gasseparation membranes, oxygen sensors, chemical looping devices, and memristors. Many of the best oxygen ion conductors are perovskites, and searching this crystal class for fast, stable, and application-compatible oxygen conductors has been an active area of research for decades.[2, 3]

Optimized vacancy content and low oxygen migration barrier are typically the dominant factors leading to fast vacancy-mediated oxygen transport in perovskites. This paper assumes that doping strategies can optimize vacancy content $[1,4]$ and instead focuses solely on oxygen migration barrier. Sections 3 and 4 discuss briefly where information about vacancy concentration and dopant association effects would be valuable additional knowledge. 
As an example of the scale of the effect of oxygen migration barrier on oxygen transport, for a hypothetical material at $1073 \mathrm{~K}\left(800^{\circ} \mathrm{C}\right)$, a decrease of $0.4 \mathrm{eV}$ in migration barrier energy corresponds to a 100 times increase in oxygen ion conductivity, potentially allowing a 100 times increase in the thickness of an SOFC electrolyte (see Supporting Information (SI), Section S2.1). Lowering the migration barrier by $0.6 \mathrm{eV}$ at the same temperature could reduce the cathode area specific resistance (ASR) by 100 times (see SI, Section S2.2). These effects could enable significantly more stable, lower-temperature, cheaper SOFCs.

Descriptors for oxygen migration barrier could help identify low barrier materials prior to synthesis efforts. Previously-proposed descriptors for oxygen migration in perovskites include Kilner critical radius,[5-8] Goldschmidt tolerance factor,[5] volumetric factors,[5] crystal structure ideality,[9] oxygen vacancy formation energy,[10,11] and metal-oxygen bond strength (fluorites [12]), both average[5] and related to vacancy trapping (summarized in Ref. [13]). This study focuses solely on perovskites, considers over 40 Asite and B-site combinations, uses oxygen migration barrier data from a single source and method, and evaluates dozens of descriptors at once, looking for a simple functional relationship between oxygen migration barrier and each proposed descriptor.

\section{Methods}

\subsection{Choosing and evaluating descriptors}

Because the descriptors are meant to be predictive, this study focuses on descriptors that do not require prior knowledge about the activated transition state. That is, we focus on descriptors from the bulk or initial defected state, and leave out descriptors which must be obtained from a transition-state calculation itself, such as transition state geometry[7] or actual path length.

Evaluating a descriptor consists of looking for the presence of a simple function, though not necessarily linear, between oxygen migration barrier and that descriptor. While combining descriptors might produce better correlations, evaluating descriptors individually provides a clear picture of the controlling physics without the risk of over-fitting.

Although fewer than two dozen descriptors appear in this study's main text and SI Section 3, we actually looked for correlation with hundreds of possible descriptors, of which only the most well-known, often-suggested, best-performing, and/or representative of key physics were included. These descriptors include those we have invented, those that have been proposed as important when comparing across crystal structures, and those that are commonly cited as being important within the perovskite crystal structure.[5, 9]

Most of the descriptors that were discarded were permutations of a single descriptor type, for example, the distance between a specific B-site cation and its neighboring oxygen in the positive $\mathrm{z}$ direction; in the negative $\mathrm{z}$-direction; and so on. 


\subsection{Choosing systems}

Compounds were generated and screened in the following order:

- $\mathrm{La}^{3+}\left[3 \mathrm{~d}\right.$ transition metal, excluding $\mathrm{Cu}$ and $\mathrm{Zn}$, plus Ga ${ }^{3+} \mathrm{O}_{3}$ due to the prevalence of $3 \mathrm{~d}$ transition metals and $\mathrm{Ga}$ in known and studied devices.

- $[\mathrm{Pr}, \mathrm{Y}]^{3+}\left[3 \mathrm{~d}\right.$ transition metal, excluding $\mathrm{Cu}$ and $\mathrm{Zn}$, plus Ga ${ }^{3+} \mathrm{O}^{3}$ : the chemical similarity but different radii of $\mathrm{Pr}^{3+}$ and $\mathrm{Y}^{3+}$ compared to each other and to $\mathrm{La}^{3+}$ allows A-site cation size effects to become evident.

- $\mathrm{La}^{3+}[\mathrm{Al}, \mathrm{In}, \mathrm{Tl}]^{3+} \mathrm{O}_{3}$ due to the B-site similarity with fast conductors with B-sites Sc and Ga.

Assorted other compounds were partially or fully evaluated. Some were chosen because of A-site similarity to $\mathrm{La}^{3+}$ and guidance from observed correlations that suggested low barrier materials, e.g., $\mathrm{SmGaO}_{3}$. Others were chosen for $4 \mathrm{~d}$ or $5 \mathrm{~d}$ transition metal B-site cations similar to those previously studied, e.g. $\mathrm{LaRuO}_{3}$. Finally, a few $\mathrm{A}^{2+} \mathrm{B}^{4+} \mathrm{O}_{3}$ systems were evaluated to check the behavior of the correlations in the $\mathrm{A}^{2+} \mathrm{B}^{4+} \mathrm{O}_{3}$ system.

\subsection{Computational Methods}

Vacancy-mediated oxygen diffusion in these systems was modeled by a single hop from oxygen position o29 to o30, which should be taken to be sampling possible hopping barriers that range over $0.4 \mathrm{eV}$ based on calculations of all hops within three supercells (see Ref.[14] electronic supplementary information (ESI) Figure S2.1 for atomic positions, and Ref. [14] ESI Section S8 and this work's SI Section S4 for the range of hops). The exception was the barrier for $\mathrm{LaRuO}_{3}$, where we believe specific geometry in that hop forced it to exceed the range of reasonable migration barrier values for the system as a whole; therefore, the 029 to 030 migration barrier $(1.847 \mathrm{eV})$ was substituted out and an in-plane migration barrier (oxygen position o31 to o30) was used instead (1.428 eV).

Climbing nudged-elastic-band calculations $[15,16]$ were automated using the MAterials Simulation Toolkit (MAST) $[17,18]$ and performed using the Vienna Ab-initio Simulation Package (VASP) [19] on a 2x2x2 formula unit supercell where internal relaxation allowed octahedral tilting,[20,21] and with settings and approaches, including pseudopotentials picked for accuracy, as described previously.[14]

GGA+U[22-25] calculations were not used in this study due to the uncertainty in selecting $\mathrm{U}$ values,[26-28] the computational expense of performing such selection for a wide variety of cations, and convergence issues in GGA+U calculations, particularly in Co and Ni. Due to the cancellation of errors between initial and activated states in migration barrier calculations, and due to the absence of any redox occurring in our models, we expect GGA calculations to be fairly accurate for the key values in this paper. The decision to use GGA methods has also been made in other perovskite migration barrier studies.[7, 11].

Because mobile oxygen vacancies in a typical host perovskite will be chargecompensated.[1,29, 30], all migration barriers discussed in the main text use a chargecompensated oxygen vacancy, where the electrons donated by the vacancy are removed 
from the system either through explicit electron-removal or through explicit Strontium doping on the A-site, as described previously.[14] (See the SI for more discussion.) Table A.1 also includes non-charge-compensated data, in which the electrons donated from the vacancy are left in the system, often to reduce a nearby B-site cation.

Vacancy formation energies were calculated as in Lee et al.[31] Where the oxygen vacancy was charge-compensated by explicit electron removal, an extra term was applied to the vacancy formation energy in order to account for the removal of those electrons to the electron reservoir of the bulk, as in Section 3.1 of Lin et al.[32] The potential alignment correction in such cases used, for each species, the mean for all atoms of that species in the supercell. These vacancy formation energies are intended for use as descriptors rather than as energy values that can be compared to experiments, and they may not be accurate for the latter. In particular, no finite-size scaling correction was applied, the errors in the GGA approximations may affect the absolute value of each vacancy formation energy,[31] and the given oxygen partial pressure and temperature, while possible in an SOFC, may not be appropriate for all systems. Furthermore, the values do not take into account the full defect chemistry model of the perovskites and therefore are in most cases not representative of experimental vacancy formation energies. For the purposes of this study, the important value is the relative difference in oxygen vacancy formation energy between different systems.

Radii for use in descriptors were picked from the Shannon crystal radii[11, 33] based on an estimate of high-spin and low-spin state from undefected bulk magnetic moment calculations, using 6-coordination for $\mathrm{O}^{2-}, \mathrm{B}^{3+}$, and $\mathrm{B}^{4+}$, and 12-coordination, or 9coordination if 12 -coordination was not available, for $\mathrm{A}^{2+}$ and $\mathrm{A}^{3+}$. For doped systems, the $\mathrm{A}$-site radius was the weighted average of the radii for the A-site occupations.

\section{Results}

This section compares the calculated barriers with experiment where applicable, then plots migration barrier versus Mendeleev number, to show the scale of the results, and then versus the two best-performing descriptors, oxygen vacancy formation energy and oxygen p-band center energy. SI Section 3 contains the results for the other descriptors.

Each oxygen hop followed a curved migration path around a B-site cation.[13, 34] Calculating all oxygen hops for three systems indicates that each hop should be considered to have been sampled from an approximately uniformly distributed range of about $0.4 \mathrm{eV}$ (see Ref. [14] ESI Section S8, and SI for this paper, Figure S4.1 and Figure S4.2). Dopant positions may also contribute to a spread of barriers, with a spread estimated by select studies to be about $0.3 \mathrm{eV}$ (see Ref.[14], ESI Section S7). Supercell size is estimated to have a non-systematic effect of approximately $0.1 \mathrm{eV}$ (see Table A.1).

Figure 1 compares our calculations to migration barriers from experimental data, whose details are given in SI Table S1.1. (For a comparison with calculated rather than experimental literature data, see SI Figure S1.2.) For each computed value there is a 
comparable experimental value within the range bar of the calculation. However, the large spread in the experimental values and the uncertainty in the calculations makes any quantitative comparison very difficult. We note that many of the non-transition-metal Bsite cations have predicted values lower than any of the experiments, and some of our predictions for this class of compounds (discussed in Section 4.3) also have very low values. Although these values are within the range bars of the experiments, they may also be lower because of dopant effects. Where the calculations were performed on undoped systems, with the vacancy compensated as though it had been created with dopants, which would be a requirement for these non-transition-metal B-site systems, [3, 4] the lack of any dopant association terms may lead to lower predicted migration barriers than in experiment. $[6,35,36]$

For $\mathrm{LaGaO}_{3}$, the calculation was also performed on a doped system but was still low compared to experiment. In this case, the range bar for doped hops may be necessary to represent all of the hops, including higher barrier ones, which might be necessary barriers to overcome to follow a percolating pathway in a real system. Also, the relatively high dopant concentration $(x=0.25)$ and relatively small calculation supercell size mean that in periodic space, the calculation has actually formed an ordered structure, which may not be comparable to a disordered structure with lower dopant concentration $(\mathrm{x}=0.1$ for LSGM[4]).

However, there is good agreement between our calculations and experiment for $\mathrm{LaScO}_{3}$, LSS, LSSM, and $\mathrm{BaTiO}_{3}$, which also fall under the same category of non-transition-metal Bsite cation. These results suggest that a detailed system-by-system comparison is necessary to identify all of the sources of differences between the systems and experiment, possibly through an exploration of different calculation parameters, for example, pseudopotential choice, supercell size, and exact doping concentration.

In summary, the primary sources of differences between the calculations and experiments may be: (1) system-specific, for example relating to the calculation parameters of a given species, and which may require in-depth system-specific studies, and (2) model-based, for example, the absence of dopants and dopant-vacancy interactions in most of the calculated systems; the high-concentration of dopants and periodic ordering of dopants in the nine calculated doped systems; the non-dilute concentration of vacancies in most of the calculated systems (1/24 oxygen sites in a $2 \times 2 \times 2$ size supercell), which would be related in experiments to the dopant concentration, B-site cation species, temperature, and oxygen partial pressure; and the use of a single hop which does not capture full information on the percolating pathway for diffusion, where long-range diffusion is controlled by the rates of single atomic hops but is the result of many such hops in series. $[37,38]$ Interrelations among all of these differences make it difficult to make an overall statement on our calculated migration barriers versus the experimental migration barriers except to say that in general, we would not expect a single hop to be directly comparable to the migration barrier generated from experimental data, so the range bars provided should be used when comparing to experimental data. 


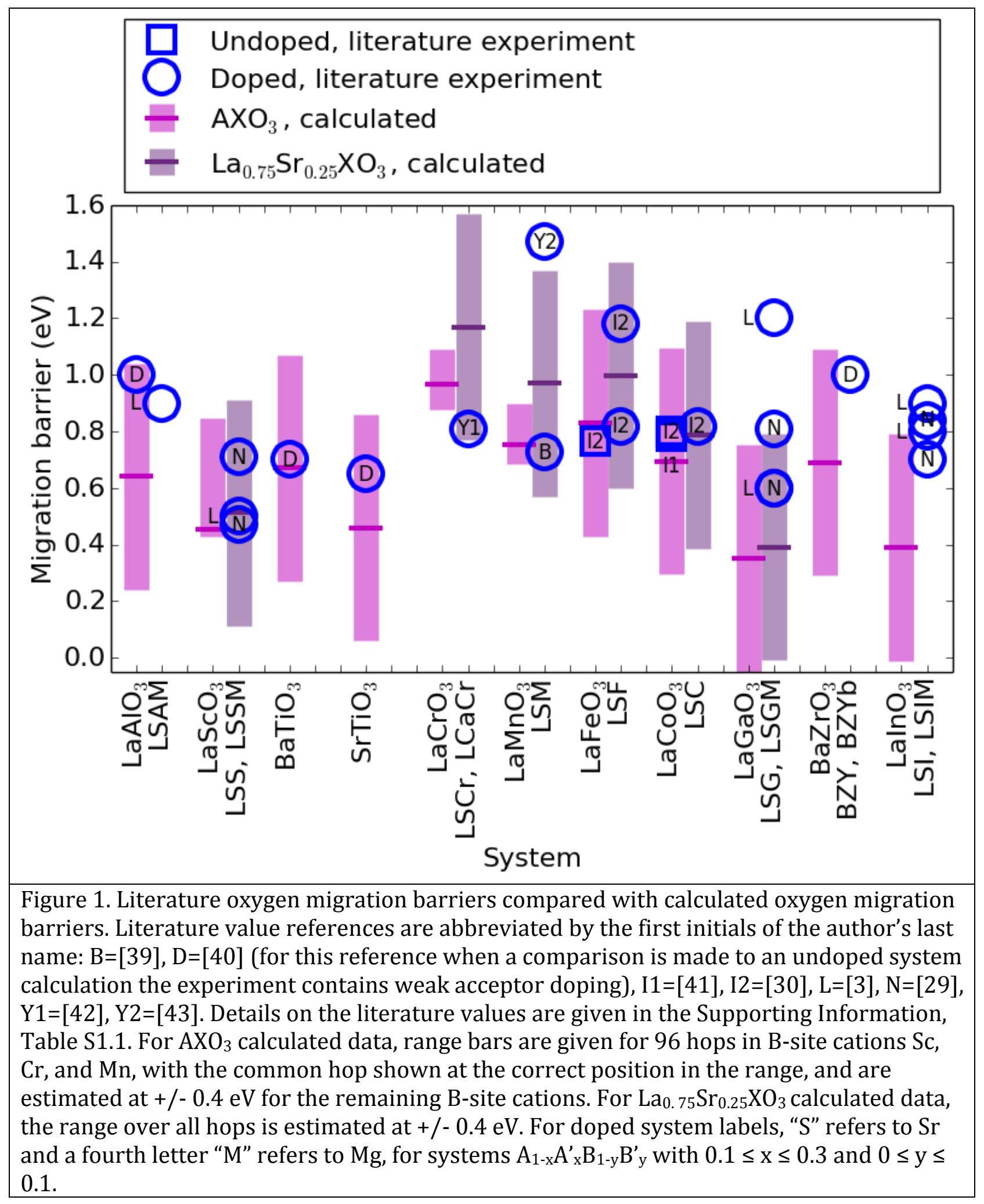

Figure 2 presents our migration barrier data plotted against a B-site cation Mendeleev number (see Figure 1 in Ref. [44]). (See SI Figure S3.A1 for the data plotted against atomic 
number, and SI Figure S3.A2 for the data plotted against a more spread-out version of Mendeleev number.) Migration barriers generally fall moving through the B-site cation Mendeleev numbers 50-81 with particularly low migration barriers calculated for nontransition-metal B-site cations ( $\mathrm{Sc}, \mathrm{Ga}, \mathrm{Al}, \mathrm{In}, \mathrm{Tl}, \mathrm{Y}$ ). More discussion on extremely low barriers will be given in Section 4, Discussion.

The calculated migration values cover a wide range of over $1.4 \mathrm{eV}$, and include some particularly low values. To understand the origin of this spread and these low values, we looked for correlation with hundreds of possible descriptors, focusing on over 20 of the most well-known or representative of key physics, as described in the Methods section. We find weak or no correlation between oxygen migration barrier and descriptors relating to space, volume, or ideality of the structure (see SI Section 3). However, we find strong correlation between oxygen migration barrier and descriptors related to metal-oxygen bond strength. A correlation of oxygen transport with metal-oxygen bond strength has been proposed several times in the literature [9, 45-47] and our large database now allows these relationships to be definitively identified. Here we focus on two measures of metaloxygen bond strength, the vacancy formation energy and oxygen p-band center energy.

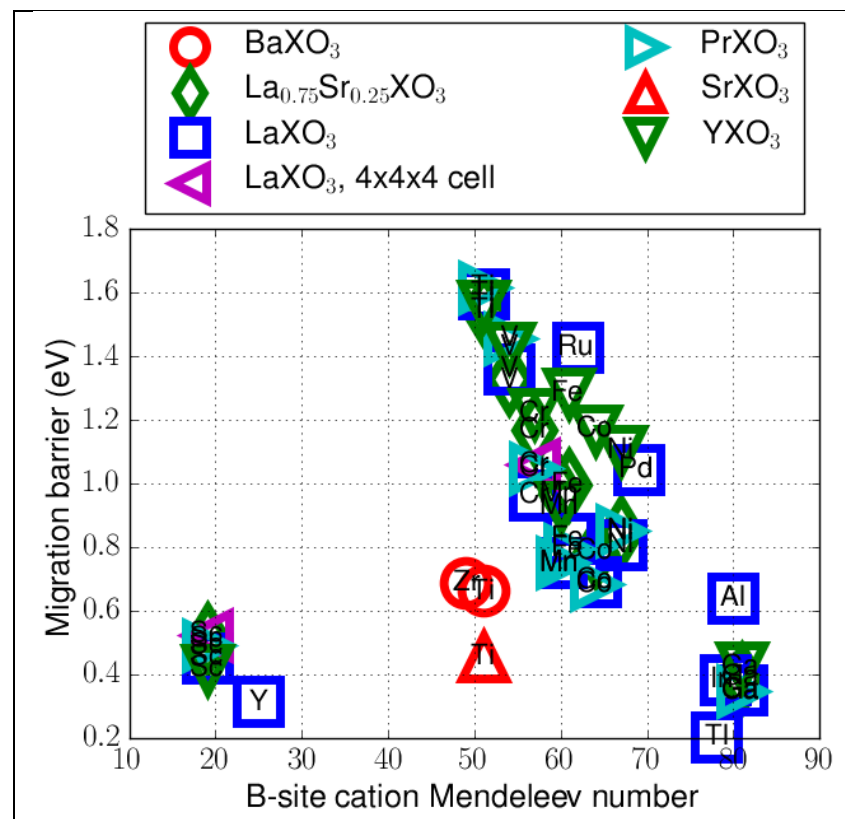

Figure 2. Oxygen migration barrier versus B-site cation Mendeleev number. Note that the red cluster at center-bottom is for $\mathrm{A}^{2+} \mathrm{B}^{4+} \mathrm{O}_{3}$ perovskites.

Figure 3 shows that oxygen vacancy formation energy strongly with oxygen migration barrier. While oxygen vacancy formation energy has been proposed as a descriptor for oxygen diffusion before, $[10,11,48]$ our data shows that the correlation holds over dozens of perovskite systems. Kwon et al. studied oxygen vacancy formation energy over a subset of B-site cations with alloying and did not note the correlation between oxygen vacancy formation and oxygen migration barrier. However, an examination of their $\mathrm{x}=0$ (undoped) 
values shows the same trends as in Figure 3 (blue squares, $\mathrm{Cr}, \mathrm{Mn}, \mathrm{Fe}, \mathrm{Co}, \mathrm{Ni}$ ), although the correlation we observe would not be apparent from just those values.[48] The root-meansquare (RMS) error between calculated migration barrier and migration barrier as a result of a linear fit with oxygen vacancy formation energy is just $0.2 \mathrm{eV}$, and also has an average of $0.2 \mathrm{eV}$ cross-validation score (with a standard deviation of $0.02 \mathrm{eV}$ ) over 1000 2-fold (leave-out-half the data, chosen randomly, for testing) cross validation tests.[49] The quite small cross-validation score and its closeness to the RMS error of the fit to all of the data strongly support the validity of the descriptor.

The observed correlation between migration and vacancy formation energy can be understood physically by noting that lower oxygen vacancy formation energy indicates that it is easier to break cation bonds with oxygen, which is a necessary part of the process for migrating an oxygen atom from one position to another. This correlation is conceptually useful, and of some practical value for computations as vacancy energies are somewhat faster to determine than migration barrier.

These oxygen vacancy formation energies are not comparable to experimentally observed oxygen vacancy formation energies, as the method used is chosen to faithfully represent the energy of breaking bonds to create the vacancy, not the often complex defect coupling that controls observed experimental vacancy formation energies. The values should therefore be taken as relative values (see Section 2.2, Computational Methods).

Furthermore, the values have a possible spread of some $+/-0.4 \mathrm{eV}$ due to the range of values introduced by symmetry-distinct oxygen sites and varied dopant positions (see SI Section S4).

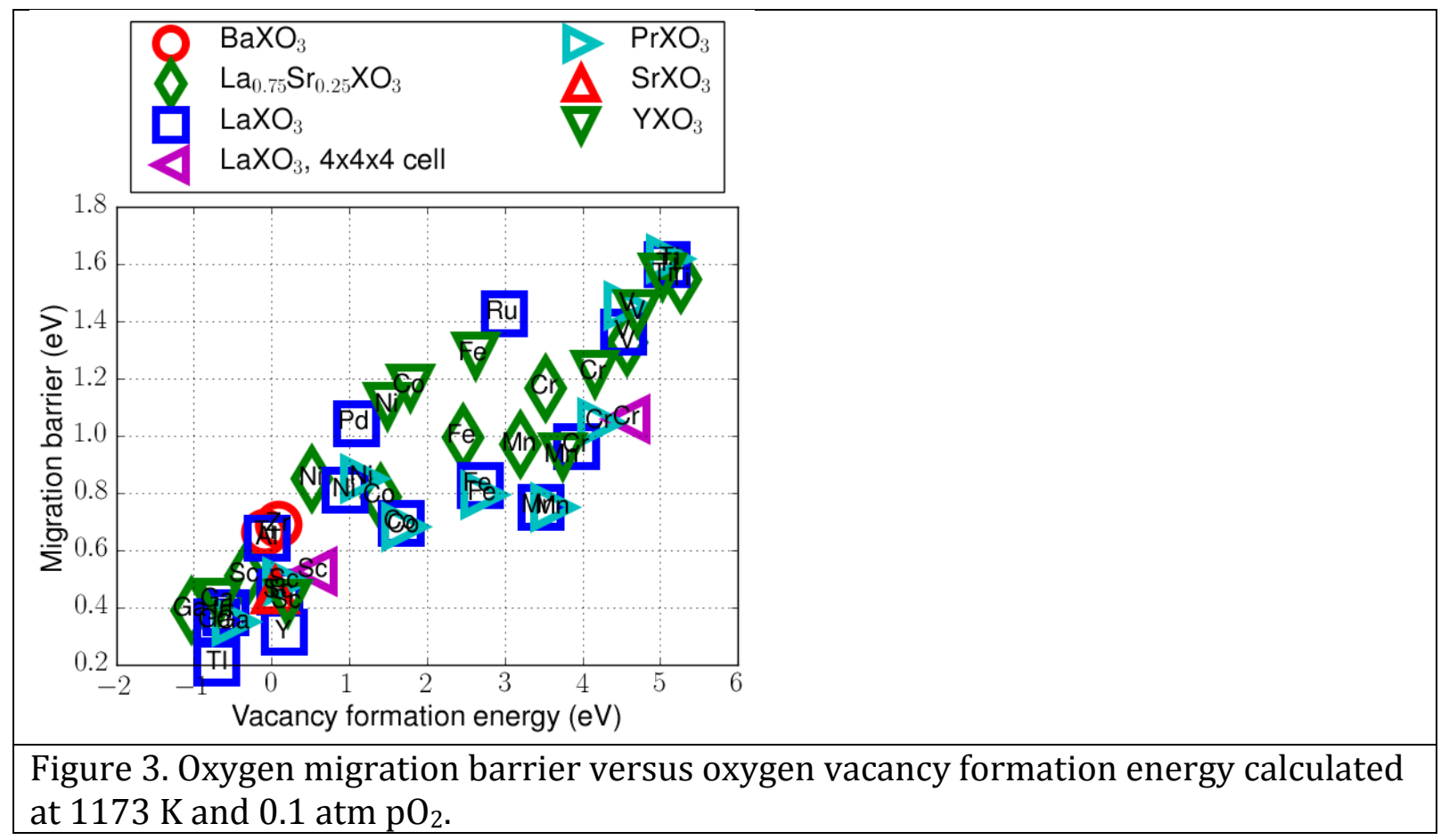


Figure 4 shows the oxygen migration energy as a function of the oxygen $2 p$-band center position relative to the Fermi level, which we abbreviate as oxygen p-band center energy. This quantity has recently been shown to correlate with many important oxygen kinetics properties in perovskites, including calculated oxygen defect and adsorption energetics[50] and experimentally measured oxygen surface exchange rates,[50] area specific resistance,[50] activation energies of surface exchange and diffusion,[51] and work functions.[52] As pointed out in Ref. [50], the oxygen p-band is also a measure of metaloxygen bond strength, as increasing p-band correlates with less charge transfer from cations to oxygen and weaker bonding between them. Therefore, the strong negative correlation shown in Figure 4 further supports the hypothesis that the oxygen migration is largely controlled by the strength of the oxygen-cation bonds.

The RMS error between calculated migration barrier and migration barrier as a result of a linear fit with oxygen p-band center is just $0.2 \mathrm{eV}$, and also has an average of $0.2 \mathrm{eV}$ crossvalidation score (with a standard deviation of $0.02 \mathrm{eV}$ ) over 10002 -fold (leave-out-half the data, chosen randomly, for testing) cross validation tests.[49] The quite small crossvalidation score and its closeness to the RMS error of the fit to all of the data strongly support the validity of the descriptor.

Note, however, that neither correlation with oxygen vacancy or p-band is particularly strong for moderate-barrier materials: a vacancy formation energy of about $3 \mathrm{eV}$ or an oxygen p-band center energy of about $-4.5 \mathrm{eV}$ span approximately $0.7 \mathrm{eV}$ of migration barriers. Therefore, these correlations may be useful more as guides than as quantitative predictors.

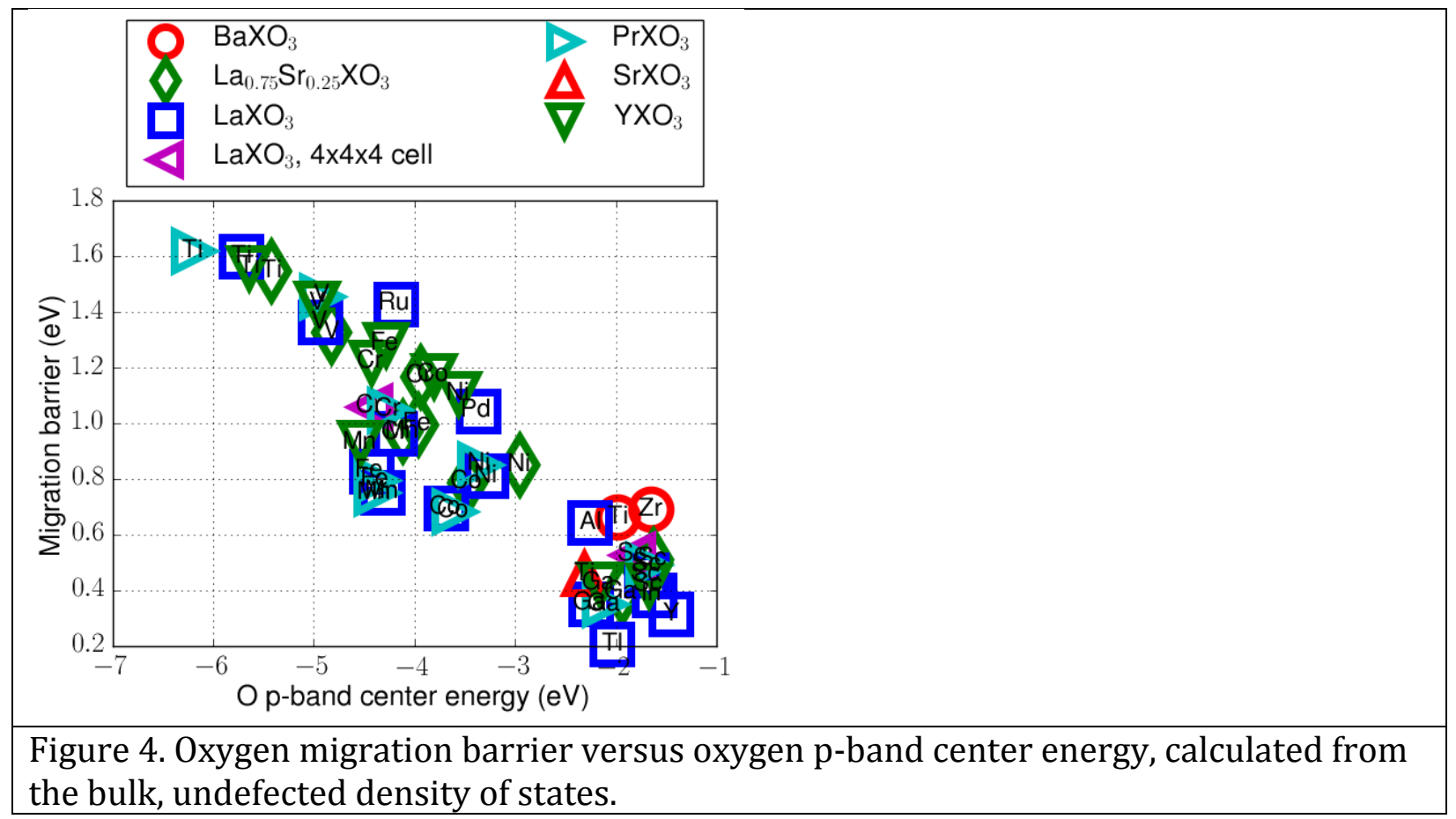




\section{Discussion}

\subsection{Oxygen $p$-band center}

The oxygen p-band center is a particularly useful descriptor as it can be rapidly calculated for a new system, making it a valuable tool for rapid screening. As the oxygen p-band correlates well with both vacancy formation energy (see SI Figure S1.1 and Refs.[50, 53]) and migration barrier (Figure 4), the oxygen p-band center energy should also correlate well with the activation energy for diffusion. This correlation has recently been shown in Ref. [51]. We therefore expect that the oxygen p-band center energy correlates well with overall diffusion coefficient $D$, although the uncertainty in overall diffusion value measurements has so far made this correlation hard to establish, as discussed in Ref. [51]. Furthermore, Lee et al. show that oxygen p-band center energy correlates with the surface exchange coefficient, $k^{*}$.[50] If the oxygen p-band correlates with $D$ and $k^{*}$, then from the Adler-Lane-Steele model (see SI Section S2.2),[54] the oxygen p-band center energy should correlate with overall cathode area-specific resistance (ASR), also consistent with what is shown in Lee et al.[50] The similar success of these many coupled correlations demonstrates the strong consistency in these correlative relationships, shows the power of this descriptor for modeling SOFCs and general active-oxygen materials, and supports the assertion that metal-oxygen bond strength plays a dominant role in the performance of these materials.

An important implication of the above results is that the fast oxygen ion conductors will typically have the weakest cation-oxygen bonds, which will make them particularly susceptible to reduction. Thus this correlation can explain the tendency of many fast oxygen ion conductors to have problems with second phase formation and increased electronic conductivity from defects when used as electrolytes exposed to reducing anodic conditions.[55]

\subsection{Defining descriptors within a scope}

We were initially surprised by the non-correlation of geometric factors over our dataset, given the emphasis on geometry in the literature. On further reflection, the finding makes sense considering, on the one hand, our large variety of A-site and B-site cations, and on the other hand, our relatively narrow constraints of using mostly $\mathrm{A}^{3+} \mathrm{B}^{3+} \mathrm{O}_{3}$ perovskites that, when doped, had only a single doping level, single dopant type, and fixed dopant positioning.

Some geometric descriptors may be more effective in a cross-class materials search, where there are dramatic changes across structure classes. For example, Sammells et al. found a $\mathrm{V}$-shaped correlation between activation energy and free volume, with a negative correlation for $\mathrm{A}^{2+} \mathrm{B}^{4+} \mathrm{O}_{3}$ perovskites and a positive correlation for fluorites[5]; our database did not have free volumes as high as those for the fluorites. Our study also looked only at single crystal diffusion, while polycrystalline diffusion and grain boundary diffusion might introduce additional geometric and volumetric factors. 
Other geometric descriptors may be more effective in a very narrow subset of systems. For example, Mogensen et al. considered structure stress to be of more importance than bond strength, but their study only spanned perovskites with non-transition-metal B-site cations in Group 3A (Sc, Ga, Al, In).[56] Similarly, SI Section S3 shows many separations by A-site cation, so looking over several A-site cations at a single B-site cation may well show trends like in Ranløv et al. (reproduced in Ref. [9] Figure 4). Structure stress, crystal structure ideality, and volume descriptors may work best when looking at fine changes in an otherwise very similar geometry.

This understanding that descriptors may apply only within a certain scope of materials helps unify our understanding of descriptors in perovskites by suggesting that there may be several best or best combinations of descriptors, each most appropriate to a specific search space and the application context. For the combinatorial problem of a single structure class with many compositions, which we undertook here, geometry may be similar enough that large changes do not dominate, while not so similar that very fine changes can be differentiated, and therefore geometric descriptors show only weak correlation. The descriptors of metal-oxygen bond strength proved the most useful for extrapolation of oxygen ionic migration barrier within the perovskite structure class, across many compositions.

\subsection{Low-barrier materials}

Several of our calculated oxygen migration barriers for non-transition-metal B-site cations are significantly below $0.5 \mathrm{eV}$ (e.g. $0.21 \mathrm{eV}, \mathrm{LaTlO}_{3}$, and $0.32 \mathrm{eV}, \mathrm{LaYO}_{3}$, see Table A.1), Low barriers are expected in the region of non-transition-metal B-site cations,[3] but no barriers this low have been reported from previous experimental studies.

Dopants may be the main reason for the discrepancy between the lower bound of the calculated migration barriers and the lower bound of migration barriers seen in experiment. While these systems lack dopants in the calculation, they are precisely the systems that usually require aliovalent dopants to enable oxygen migration. The extremely low calculated migration barriers might then be regarded as an ideal lower-bound barrier for oxygen conduction, in a system where the moving vacancy is compensated as though it were created by aliovalent dopants, but is not hampered by dopant-vacancy interactions.

Significant previous work has been done to reduce dopant-vacancy association energies, notably through the host-dopant size studies.[6, 9] One strategy to eliminate dopantvacancy association might be to form vacancies in nearby layers of doped or undoped transition metal B-site perovskites. Figure 3 shows that non-transition-metal B-site perovskites have the lowest compensated vacancy formation energies, so oxygen vacancies may collect in these materials from neighboring materials in which the vacancies were created. Epitaxial strain could also be used to induce preferential vacancy segregation into the low-barrier material.[57] These and other strategies, combined with advanced 
materials growth and deposition technologies could yield exceptional fast oxygen ion conductors in this class of non-transition-metal B-site cation perovskites.

\section{Conclusions}

We find that a database of $\mathrm{A}^{3+} \mathrm{B}^{3+} \mathrm{O}_{3}$ (and select $\mathrm{A}^{2+} \mathrm{B}^{4+} \mathrm{O}_{3}$ ) perovskites relevant for oxygen ion transport and exchange applications have calculated oxygen migration barriers ranging from 0.2 to $1.6 \mathrm{eV}$, with the lowest barrier values calculated for $3+$ cations on the B-site (Sc, $\mathrm{Al}, \mathrm{Ga}, \mathrm{In}, \mathrm{Tl})$. Notably, low barriers were predicted from our trends and found for $\mathrm{LaTlO}_{3}$ and $(\mathrm{Sm}, \mathrm{Dy}, \mathrm{Er})(\mathrm{Al}, \mathrm{Ga}) \mathrm{O}_{3}$. Such low barriers may only be accessible without significant dopant-vacancy interaction and their use as an estimate of the overall oxygen vacancy diffusion barrier is subject to significant uncertainty. Although these systems are also likely to require doping in order to produce a significant amount of vacancies, the low barriers may be accessible if such materials could be created without vacancies. The magnitude of the range and the physics of the migration appear to be dominated by metal-oxygen bond strength, as measured by oxygen vacancy formation and oxygen p-band center energy. Other descriptors relating to space, volume, and ideality of the structure do not correlate well with oxygen migration barrier in this dataset.

\section{Acknowledgments}

The National Science Foundation (NSF) Graduate Fellowship Program under Grant No. DGE-0718123 and the UW-Madison Graduate Engineering Research Scholars Program provided funding for T. Mayeshiba. Calculations utilized computing resources of the Extreme Science and Engineering Discovery Environment (XSEDE), which is supported by NSF grant number OCI-1053575, and from the UW-Madison Center for High Throughput Computing (CHTC). Support for D. Morgan and for the MAST tools applied in this work were provided by the NSF Software Infrastructure for Sustained Innovation $\left(\mathrm{SI}^{2}\right)$ award No. 1148011. T. Mayeshiba would like to thank Dr. Henry Wu for fruitful discussion, and Dr. Glen Jenness for sharing his oxygen p-band center energy code.

Supporting Information. Notes for literature values, figure for oxygen p-band energy versus oxygen vacancy formation energy, derivation of the effect of oxygen migration barrier on oxygen conductivity and cathodic ASR, and figures for additional descriptors, including Goldschmidt tolerance factor, Kilner critical radius, percent free volume, cohesive energy, Fermi energy, and bandgap, among many others. 


\section{Appendix}

Table A.1. Complete table of oxygen migration barrier data calculated for perovskite systems, including data for charge-compensated and non-charge compensated vacancies. See Section 2, Computational Methods and SI Section S1 for an explanation of compensation. Italics denote an $\mathrm{A}^{2+} \mathrm{B}^{4+} \mathrm{O}_{3}$ perovskite; all others are $\mathrm{A}^{3+} \mathrm{B}^{3+} \mathrm{O}_{3}$ or $\mathrm{A}^{3+}{ }_{0.75} \mathrm{~A}^{\prime 2+}{ }_{0.25} \mathrm{~B}^{3+} \mathrm{O}_{3}$. Systems evaluated for low migration barrier based on correlations may be missing some data, as they were not part of the original calculation set.

\begin{tabular}{|c|c|c|c|c|c|c|}
\hline \multirow[b]{2}{*}{ System } & \multicolumn{2}{|c|}{$\begin{array}{l}\text { Oxygen migration } \\
\text { barrier }(\mathrm{eV})\end{array}$} & \multicolumn{2}{|c|}{$\begin{array}{l}\text { Oxygen } 2 \text { p-band } \\
\text { center energy }(\mathrm{eV})\end{array}$} & \multicolumn{2}{|c|}{$\begin{array}{c}\text { Oxygen vacancy } \\
\text { formation energy } \\
(\mathrm{eV}) \text { at } 1173 \mathrm{~K}, 0.1 \\
\text { atm } \mathrm{p}\left(\mathrm{O}_{2}\right)\end{array}$} \\
\hline & $\begin{array}{l}\text { Charge- } \\
\text { compen- } \\
\text { sated }\end{array}$ & $\begin{array}{l}\text { Non- } \\
\text { charge- } \\
\text { compen- } \\
\text { sated }\end{array}$ & $\begin{array}{l}\text { Charge- } \\
\text { compen- } \\
\text { sated }\end{array}$ & $\begin{array}{l}\text { Non- } \\
\text { charge- } \\
\text { compen- } \\
\text { sated }\end{array}$ & $\begin{array}{l}\text { Charge- } \\
\text { compen- } \\
\text { sated }\end{array}$ & $\begin{array}{l}\text { Non- } \\
\text { charge- } \\
\text { compen- } \\
\text { sated }\end{array}$ \\
\hline $\mathrm{BaTiO}_{3}$ & 0.67 & & -1.99 & & -0.09 & \\
\hline $\mathrm{BaZrO}_{3}$ & 0.69 & & -1.66 & & 0.08 & \\
\hline $\mathrm{DyAlO}_{3}$ & 0.55 & & -2.30 & & 0.04 & \\
\hline $\mathrm{DyGaO}_{3}$ & 0.44 & & -2.09 & & -0.65 & \\
\hline $\mathrm{ErAlO}_{3}$ & 0.59 & & -2.33 & & 0.11 & \\
\hline $\mathrm{ErCoO}_{3}$ & 1.22 & & -3.98 & & 1.08 & \\
\hline $\mathrm{ErGaO}_{3}$ & 0.47 & & -2.05 & & -0.62 & \\
\hline $\mathrm{La}_{0.75} \mathrm{Sr}_{0.25} \mathrm{CoO}_{3}$ & 0.79 & & -3.48 & & 1.40 & \\
\hline $\mathrm{La}_{0.75} \mathrm{Sr}_{0.25} \mathrm{CrO}_{3}$ & 1.17 & & -3.95 & & 3.52 & \\
\hline $\mathrm{La}_{0.75} \mathrm{Sr}_{0.25} \mathrm{FeO}_{3}$ & 1.00 & & -3.96 & & 2.46 & \\
\hline $\mathrm{La}_{0.75} \mathrm{Sr}_{0.25} \mathrm{GaO}_{3}$ & 0.39 & & -1.94 & & -1.04 & \\
\hline $\mathrm{La}_{0.75} \mathrm{Sr}_{0.25} \mathrm{MnO}_{3}$ & 0.97 & & -4.12 & & 3.20 & \\
\hline $\mathrm{La}_{0.75} \mathrm{Sr}_{0.25} \mathrm{NiO}_{3}$ & 0.85 & & -2.96 & & 0.51 & \\
\hline $\mathrm{La}_{0.75} \mathrm{Sr}_{0.25} \mathrm{ScO}_{3}$ & 0.51 & & -1.64 & & -0.34 & \\
\hline
\end{tabular}




\begin{tabular}{|c|c|c|c|c|c|c|}
\hline $\mathrm{La}_{0.75} \mathrm{Sr}_{0.25} \mathrm{TiO}_{3}$ & 1.55 & & -5.42 & & 5.26 & \\
\hline $\mathrm{La}_{0.75} \mathrm{Sr}_{0.25} \mathrm{VO}_{3}$ & 1.33 & & -4.83 & & 4.57 & \\
\hline $\mathrm{LaAlO}_{3}$ & 0.64 & & -2.26 & & -0.06 & \\
\hline $\mathrm{LaCoO}_{3}$ & 0.70 & 0.76 & -3.69 & -3.73 & 1.66 & 1.97 \\
\hline $\mathrm{LaCrO}_{3}$ & 0.97 & 1.67 & -4.19 & -4.24 & 3.92 & 4.29 \\
\hline $\begin{array}{c}\mathrm{LaCrO}_{3}, 4 \times 4 \times 4 \\
\text { supercell }\end{array}$ & 1.06 & 1.66 & -4.44 & -4.44 & 4.57 & 4.29 \\
\hline $\mathrm{LaFeO}_{3}$ & 0.83 & 0.81 & -4.43 & -4.49 & 2.67 & 2.66 \\
\hline $\mathrm{LaGaO}_{3}$ & 0.35 & 2.07 & -2.25 & -1.95 & -0.71 & 4.19 \\
\hline $\mathrm{LaInO}_{3}$ & 0.39 & & -1.63 & & -0.60 & \\
\hline $\mathrm{LaMnO}_{3}$ & 0.75 & 0.94 & -4.32 & -4.42 & 3.46 & 3.43 \\
\hline $\mathrm{LaNiO}_{3}$ & 0.81 & 0.90 & -3.29 & -3.29 & 0.94 & 1.22 \\
\hline $\mathrm{LaPdO}_{3}$ & 1.04 & & -3.37 & & 1.08 & \\
\hline $\mathrm{LaRuO}_{3}$ & 1.43 & & -4.18 & & 2.99 & \\
\hline $\mathrm{LaScO}_{3}$ & 0.46 & 1.97 & -1.70 & -1.70 & 0.10 & 5.59 \\
\hline $\begin{array}{c}\mathrm{LaScO}_{3}, 4 \times 4 \times 4 \\
\text { supercell }\end{array}$ & 0.53 & 1.86 & -1.84 & -1.84 & 0.54 & 5.58 \\
\hline $\mathrm{LaTiO}_{3}$ & 1.60 & 1.61 & -5.73 & -5.76 & 5.09 & 5.05 \\
\hline $\mathrm{LaTlO}_{3}$ & 0.21 & & -2.04 & & -0.71 & \\
\hline $\mathrm{LaVO}_{3}$ & 1.36 & 1.63 & -4.94 & -5.09 & 4.52 & 4.62 \\
\hline $\mathrm{LaYO}_{3}$ & 0.32 & & -1.45 & & 0.15 & \\
\hline $\mathrm{PrCoO}_{3}$ & 0.68 & 0.76 & -3.61 & -3.61 & 1.71 & 2.04 \\
\hline $\mathrm{PrCrO}_{3}$ & 1.05 & 2.00 & -4.25 & -4.25 & 4.23 & 4.33 \\
\hline $\mathrm{PrFeO}_{3}$ & 0.80 & 0.83 & -4.38 & -4.38 & 2.73 & 2.70 \\
\hline $\mathrm{PrGaO}_{3}$ & 0.35 & 2.13 & -2.11 & -2.11 & -0.48 & 4.26 \\
\hline $\mathrm{PrMnO}_{3}$ & 0.75 & 1.03 & -4.39 & -4.39 & 3.63 & 3.48 \\
\hline $\mathrm{PrNiO}_{3}$ & 0.85 & 0.88 & -3.35 & -3.35 & 1.17 & 1.40 \\
\hline $\mathrm{PrScO}_{3}$ & 0.49 & 2.03 & -1.69 & -1.69 & 0.17 & 5.51 \\
\hline $\mathrm{PrTiO}_{3}$ & 1.62 & 1.54 & -6.20 & -6.20 & 5.11 & 4.86 \\
\hline $\mathrm{PrVO}_{3}$ & 1.46 & 1.75 & -4.92 & -4.92 & 4.57 & 4.62 \\
\hline $\mathrm{SmAlO}_{3}$ & 0.55 & & -2.25 & & -0.13 & \\
\hline $\mathrm{SmCuO}_{3}$ & 0.66 & & -2.99 & & 0.23 & \\
\hline $\mathrm{SmGaO}_{3}$ & 0.36 & & -2.00 & & -0.56 & \\
\hline $\mathrm{SrTiO}_{3}$ & 0.46 & & -2.32 & & 0.05 & \\
\hline $\mathrm{YCoO}_{3}$ & 1.17 & 1.47 & -3.81 & -3.81 & 1.78 & 1.92 \\
\hline $\mathrm{YCrO}_{3}$ & 1.22 & 2.16 & -4.43 & -4.43 & 4.16 & 4.45 \\
\hline $\mathrm{YFeO}_{3}$ & 1.29 & 0.97 & -4.28 & -4.28 & 2.62 & 2.65 \\
\hline $\mathrm{YGaO}_{3}$ & 0.43 & 2.33 & -2.16 & -2.16 & -0.68 & 4.34 \\
\hline $\mathrm{YMnO}_{3}$ & 0.93 & 1.26 & -4.54 & -4.54 & 3.74 & 3.51 \\
\hline $\mathrm{YNiO}_{3}$ & 1.11 & 1.22 & -3.57 & -3.57 & 1.49 & 1.55 \\
\hline $\mathrm{YScO}_{3}$ & 0.42 & 1.90 & -1.68 & -1.68 & 0.20 & 5.59 \\
\hline $\mathrm{YTiO}_{3}$ & 1.56 & 1.57 & -5.64 & -5.64 & 5.02 & 5.13 \\
\hline $\mathrm{YVO}_{3}$ & 1.43 & 1.90 & -4.97 & -4.97 & 4.70 & 4.74 \\
\hline
\end{tabular}




\section{References}

[1] P. Knauth, H.L. Tuller, J Am Ceram Soc 85 (2002) (7) 1654.

[2] T. Takahashi, H. Iwahara, Energy Conversion 11 (1971) 105.

[3] D. Lybye, F.W. Poulsen, M. Mogensen, Solid State lonics 128 (2000) (1-4) 91.

[4] T. Ishihara, H. Matsuda, Y. Takita, J. Am. Chem. Soc. 116 (1994) 3801.

[5] A.F. Sammells, R.L. Cook, J.H. White, J.J. Osborne, R.C. MacDuff, Solid State lonics 52 (1992) 111.

[6] J.A. Kilner, R.J. Brook, Solid State lonics 6 (1982) 237.

[7] Y.A. Mastrikov, R. Merkle, E.A. Kotomin, M.M. Kuklja, J. Maier, Physical chemistry chemical physics : PCCP 15 (2013) (3) 911.

[8] J. Richter, Monatshefte fuer Chemie (2009) 985.

[9] M. Mogensen, D. Lybye, N. Bonanos, P.V. Hendriksen, F.W. Poulsen, Solid State lonics 174 (2004) 279.

[10] M. Pavone, A.M. Ritzmann, E.A. Carter, Energy \& Environmental Science 4 (2011) 4933.

[11] R. Merkle, Y.a. Mastrikov, E.a. Kotomin, M.M. Kuklja, J. Maier, Journal of The Electrochemical Society 159 (2012) B219.

[12] E.D. Wachsman, ECS Transactions 25 (2009) (2) 783.

[13] L. Malavasi, C.A.J. Fisher, M.S. Islam, Chem Soc Rev 39 (2010) 4370.

[14] T. Mayeshiba, D. Morgan, Physical chemistry chemical physics : PCCP 17 (2015) (4) 2715.

[15] G. Henkelman, B.P. Uberuaga, H. Jónsson, The Journal of Chemical Physics 113 (2000) (22) 9901.

[16] G. Henkelman, H. Jónsson, J Chem Phys 113 (2000) (22) 9978.

[17] T. Angsten, T. Mayeshiba, H. Wu, D. Morgan, New Journal of Physics 16 (2014) (1) 015018.

[18] MAterials Simulation Toolkit (MAST), (2010-2016).

[19] J. Hafner, G. Kresse, D. Vogtenhuber, M. Marsman, Vienna Ab-initio Simulation Package.

[20] A.M. Glazer, Acta Crystallographica Section B Structural Crystallography and Crystal Chemistry 28 (1972) 3384.

[21] P.V. Balachandran, J.M. Rondinelli, Physical Review B 88 (2013) (5).

[22] S.L. Dudarev, G.A. Botton, S.Y. Savrasov, C.J. Humphreys, A.P. Sutton, Physical

Review B 57 (1998) (3) 1505.

[23] A.I. Liechtenstein, V.I. Anisimov, J. Zaanen, Physical Review B 52 (1995) (8) R5467.

[24] O. Bengone, M. Alouani, P. Blöchl, J. Hugel, Physical Review B 62 (2000) (24) 16392.

[25] A. Rohrbach, J. Hafner, G. Kresse, Journal of Physics: Condensed Matter 15 (2003) (6)

979.

[26] E. Finazzi, C. Di Valentin, G. Pacchioni, A. Selloni, J Chem Phys 129 (2008) (15) 154113.

[27] S. Okatov, A. Poteryaev, A. Lichtenstein, Europhys. Lett. 70 (2005) 499.

[28] L. Wang, T. Maxisch, G. Ceder, Physical Review B 73 (2006) (19).

[29] K. Nomura, S. Tanase, Solid State lonics 98 (1997) (3-4) 229.

[30] T. Ishigaki, S. Yamauchi, K. Kishio, J. Mizusaki, K. Fueki, Journal of Solid State Chemistry 73 (1988) (1) 179. 
[31] Y.-L. Lee, J. Kleis, J. Rossmeisl, D. Morgan, Physical Review B 80 (2009) (22).

[32] S.-k. Lin, C.-k. Yeh, B. Puchala, Y.-L. Lee, D. Morgan, Computational Materials Science 73 (2013) 41.

[33] R.D. Shannon, Acta Crystallographica A32 (1976) 751.

[34] M. Cherry, M.S. Islam, C.R.A. Catlow, Journal of Solid State Chemistry 118 (1995) 125.

[35] J.A. Van Orman, K.L. Crispin, Reviews in Mineralogy and Geochemistry 72 (2010) (1)

757.

[36] B.C.H. Steele, Materials Science and Engineering: B 13 (1992) 79.

[37] R.W. Balluffi, S.M. Allen, W.C. Carter, (2005) 41.

[38] P.G. Shewmon, Diffusion in Solids, McGraw-Hill, New York (1963).

[39] A. Belzner, T. Gur, R. Huggins, Solid State lonics 57 (1992) (3-4) 327.

[40] R.A. De Souza, Advanced Functional Materials 25 (2015) (40) 6326.

[41] T. Ishigaki, S. Yamauchi, J. Mizusaki, K. Fueki, H. Tamura, Journal of Solid State Chemistry 54 (1984) (1) 100.

[42] I. Yasuda, M. Hishinuma, Journal of Solid State Chemistry 115 (1995) (1) 152.

[43] I. Yasuda, M. Hishinuma, Proceedings of the Second International Symposium on Ionic and Mixed Conducting Ceramics 94-12 (1994) 209.

[44] S. Ranganathan, a. Inoue, Acta Materialia 54 (2006) 3647.

[45] B. Yildiz, MRS Bulletin 39 (2014) (02) 147.

[46] A. Chroneos, B. Yildiz, A. Tarancón, D. Parfitt, J.A. Kilner, Energy \& Environmental Science 4 (2011) (8) 2774.

[47] A.B. Munoz-Garcia, A.M. Ritzmann, M. Pavone, J.A. Keith, E.A. Carter, Acc Chem Res 47 (2014) (11) 3340.

[48] H. Kwon, J. Park, B.-K. Kim, J.W. Han, Journal of the Korean Ceramic Society 52 (2015) (5) 331.

[49] M. Stone, Journal of the Royal Statistical Society. Series B (Methodological) 36 (1974) (2) 111.

[50] Y.-L. Lee, J. Kleis, J. Rossmeisl, Y. Shao-Horn, D. Morgan, Energy \& Environmental Science 4 (2011) (10) 3966.

[51] Y.L. Lee, D. Lee, X.R. Wang, H.N. Lee, D. Morgan, Y. Shao-Horn, J Phys Chem Lett 7 (2016) (2) 244.

[52] R. Jacobs, J. Booske, D. Morgan, Advanced Functional Materials (2016).

[53] A.M. Deml, V. Stevanović, C.L. Muhich, C.B. Musgrave, R. O'Hayre, Energy \& Environmental Science 7 (2014) (6) 1996.

[54] S.B. Adler, J.A. Lane, B.C.H. Steele, Journal of The Electrochemical Society 143 (1996) (11) 3554.

[55] M. Yano, A. Tomita, M. Sano, T. Hibino, Solid State lonics 177 (2007) 3351.

[56] M. Mogensen, D. Lybye, N. Bonanos, P. Hendriksen, F. Poulsen, Solid State Ionics 174 (2004) (1-4) 279.

[57] D.S. Aidhy, W.J. Weber, Journal of Materials Research 31 (2015) (01) 2. 